\title{
Geomagnetism, volcanoes, global climate change, and predictability A progress report
}

\author{
Giovanni P. Gregori \\ Istituto di Fisica dell'Atmosfera, C.N.R., Roma, Italy \\ World Laboratory (HEFEST Project)
}

\begin{abstract}
A model is investigated, by which the encounters of the solar system with dense interstellar clouds ought to trigger either geomagnetic field reversals or excursions, that produce extra electric currents within the Earth dynamo, that cause extra Joule's heating, that supplies volcanoes and endogenous processes. Volcanoes increase the Earth degassing into the atmosphere, hence the concentration of the minor atmospheric constituents, including the greenhouse gases, hence they affect climate temperature, glacier melting, sea level and global change. This investigation implies both theoretical studies and observational data handling on different time scales, including present day phenomena, instrumental data series, historical records, proxy data, and geologi$\mathrm{cal}$ and palaeontological evidences. The state of the art is briefly outlined, mentioning some already completed achievements, investigations in progress, and future perspectives.
\end{abstract}

Key words geomagnetism - dynamo - secular variation - inversions and excursions - tide - induction - Earth's interior - Earth's rotation - vulcanism - volcanic cycles - geothermal flow - interstellar clouds - greenhouse gases - temperature climate - sea level

\section{Introduction}

The standard interpretation of the solarterrestrial relations is in terms of a chain of causes and effects summarized by the sketch in fig. 1. Instead the interpretation envisaged here relies on the scheme depicted in fig. 2 . This is a progress report updated April 1994. For the sake of brevity, in general only a very restricted set of literature is explicitly quoted. More extensive details are given mainly in Gregori (1993), plus a few papers suitably referred to in the following. After working out this scheme, it was found that the basic idea is not new. On the time scale of several $100 \mathrm{Ma}$, the $\mathrm{CO}_{2}$ injection into the atmosphere from volcanoes was claimed by Ronov (1975) to be the essential factor that makes life survive on Earth: volcanoes are the source, and life is the sink of $\mathrm{CO}_{2}$.

It is proposed that the Earth's dynamo, while acquiring its main energy-input from the tidal pull, is modulated by the time-varying electric currents that flow within the mantle and that are induced by the solar wind, with a period longer than several years. The inner (solid) core of the Earth is supposed to rotate much faster than the mantle plus lithosphere, due to the fact that the outer (fluid) core has a very low viscosity, while the lunar tidal pull is much stronger on the mantle (plus lithosphere) than on the inner core. This implies that a dynamo is operative, by which the «static» field is given by the mantle plus lithospheric and crustal fields (including the effect of the induced telluric currents), while the moving coil is composed of the inner core and the MHD fluid of the outer core. The time varying solar 


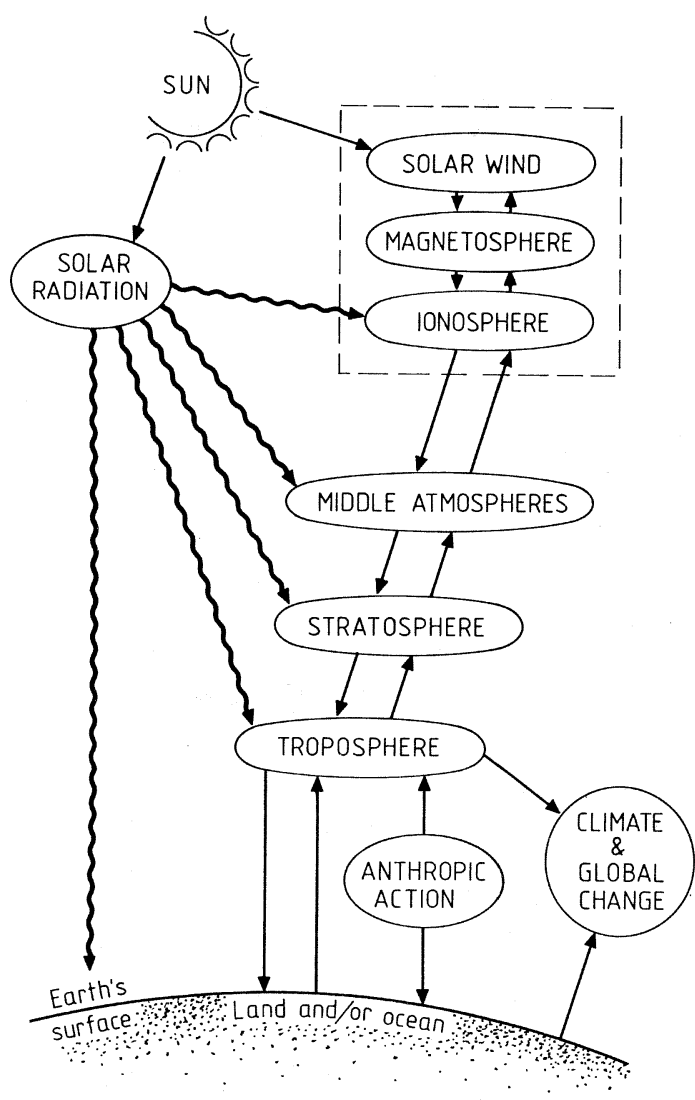

Fig. 1. Sketch showing the usual way of conceiving the chain of causes and effects that explain the solar-terrestrial relations.

induction is thus likened to a modulation of the intensity of the «static» field.

The kinetic energy input (tidal pull) can be evaluated, and is ultimately to be released mainly as Joule's heating, that proves to be conspicuous. It must be released either through volcanoes, or through geothermal heat flow, or through the thermal expansion of the Earth, and its subsequent re-shaping by gravity sliding. Hence, since there is a secular variation (SV) of the geomagnetic field, one must expect a corresponding SV both of the volcanic activity and/or of the geothermal heat flow. Moreover, one should expect that the geographic distribution of volcanoes ought to be correlated with the spatial distribution of the geomagnetic field.

Lastly, a variation in time of vulcanism and/ or of geothermal heat flow, averaged over the entire globe and integrated over some suitable time lag, ought to be associated with a timevarying degassing from either soil, and/or seas and oceans, and/or volcanoes, thus changing in time the total average concentration of the minor atmospheric constituents, hence affecting the greenhouse effect, the albedo, the ozone layer, all atmospheric chemistry, and the global climate change.

\section{Geomagnetic evidence, the dynamo, the deep Earth's structure}

Several proposals in the past were concerned with the trend of Gauss' elements of terrestrial magnetism $\left\{g_{n}^{m}, h_{n}^{m}\right\}$ vs. their degree $n$. The best such dependence seems to be Lowes' and Nevanlinna's laws, dealing with the logarithm of the average magnetic energydensity at sea level. Such a logarithm displays 3 linear trends, one for the terms $n=1$ and 2 (Nevanlinna's), one for the terms $n=3, \ldots, 13$ (Lowes'), and one for the terms $n=14, \ldots$ (Lowes'). Such three lines seem to correspond to three layers having a relevant concentration of electric currents located, respectively, at the inner-core boundary (ICB), at the core-mantle boundary (CMB), and at the base of the lithosphere, that can be called asthenosphere-lithosphere boundary (ALB) as for Gregori (1993). The dynamo here considered has no intrinsic contradiction with the previous reported MHD dynamos, the new aspect being rather concerned only with its prime energy source.

The heat transport between the ICB and the $\mathrm{CMB}$ occurs by convection within the almost frictionless fluid of the outer core, while, within mantle and lithosphere, neither conduction nor convection can afford to transport such large amounts of heat. For such a purpose, Gregori (1993) proposed, after excluding all other apparent possibilities, the existence of «pipelines» or «dykes», composed of regions having a higher electrical conductivity compared to their respective surrounding medium. 


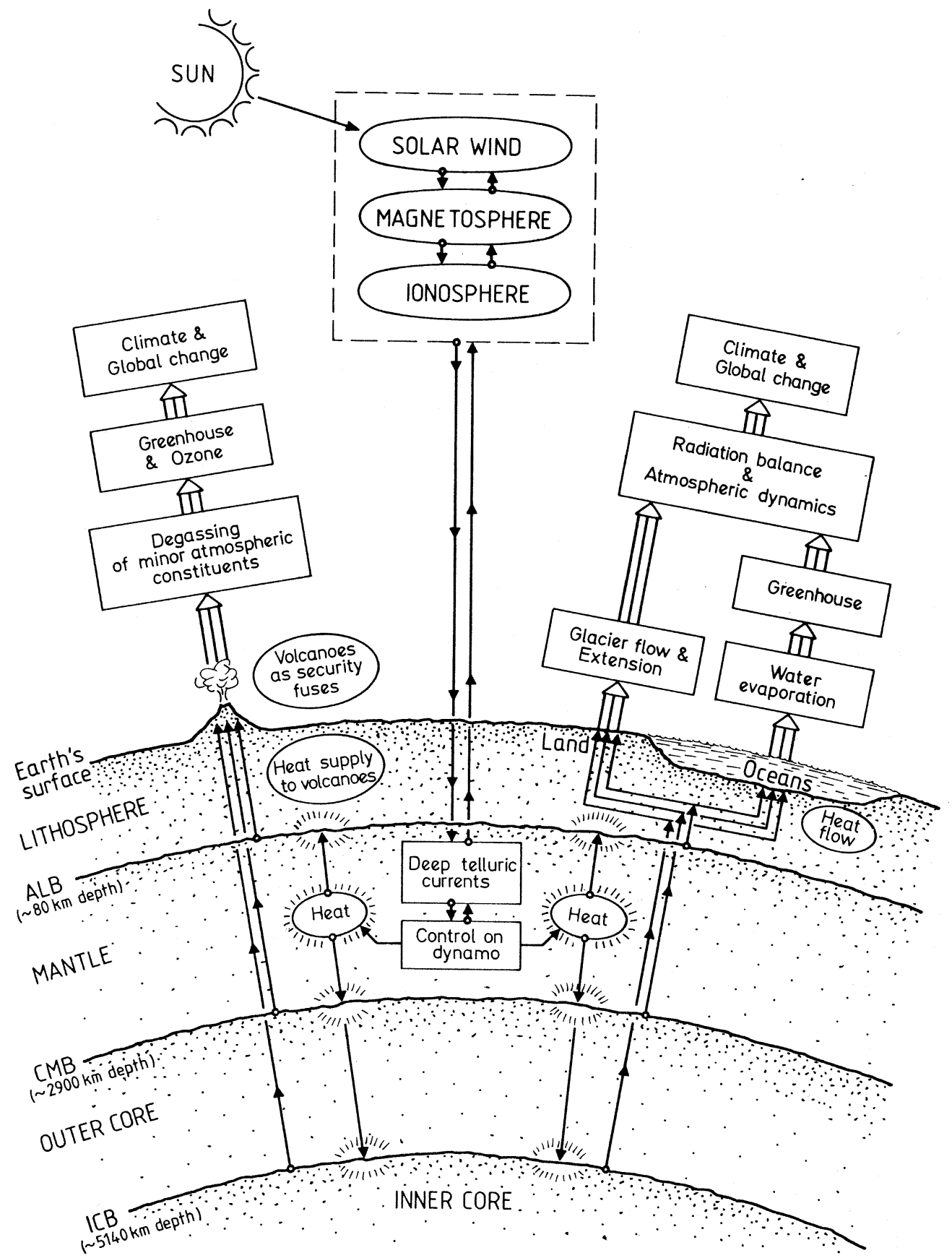

Fig. 2. Sequence of causes and effects here considered. The radiative mechanisms shown in fig. 1 ought also to be included in this scheme. 
Such «pipelines» or «dykes» ought to be produced due to the action of an outward push by the electric currents, depending on Hamilton's variational principle, much like an "electric soldering iron» pushing and penetrating into a block of ice (fig. 3). Concerning the possible simultaneous (and often speculated) existence of convection within the mantle, see below.

The investigation of the long-period currents induced in the mantle was already almost completed (in cooperation with A. Caserta and G. de Franceschi), including the estimate of the average depth of the currents (the computation was based on a variational and energy-balance principle). The most important components were found to be the first 7 harmonics of the $\sim 22$ year wave, with minor contributions also from the 8th and 9th ones. The Earth seems to resonate on the 6th harmonic.

The geographic distribution of Joule's heating generated over either one of the three layers (ICB, CMB or ALB) is being investigated (in cooperation with V. Banzon and R. Leonardi). A clear and unambiguous result was found for the ICB, where a large «stove» seems to supply heat to the Hawaiian hot spot. Hence, one must expect a stable 4-cell convective pattern within the outer core with an upwelling plume just underneath the Hawaiian hot spot, while some corresponding signature of a smaller intensity should be found at its approximately antipodean region, that perhaps can be identified with the well known «Dupal anomaly» in the isotopic chemistry of basalts (e.g. Wilson, 1989).

Another inference is related to the motion (in latitude and longitude) of the point of maximum Joule's heating that is generated on the ICB. Such an effect has been mapped since A.D. 1550 (computation in progress) on the basis of the spherical harmonic expansions (S.H.E.) of the field potential, computed at different epochs and available from the literature. But, until A.D. 1800, such S.H.E.'s are given apart an arbitrary multiplication factor, due to the fact that no total intensity measurements of the geomagnetic field were available prior to Gauss' times. It is expected, however, that both the position of the maximum (that does not depend on such an arbitrariness), and its actual peak value (that depends on it, but that can be correctly estimated from $\sim$ A.D. 1800 onward) could depend, perhaps, on solar activity. If this inference is correct, it will be possible to estimate also the value of the afore-mentioned arbitrary constant, simply by means of a solar activity index (in progress).

\section{Volcanoes and the time modulation of their activity}

The time modulation of the output from a given volcano can be evidenced by means of Imbò's algorithm (see Gregori et al., 1992, 1994). Consider a chronological list of the eruptions of a given volcano (the list can eventually contain even some gaps). Every eruption has its own order-number $j$. Associate with it the total time lag $\Delta t_{j}$ lasting between that given $j$-th eruption and the subsequent $j+1$-st one. The plot of $\Delta t_{j}$ vs. $j$ (called Imbò's histogram) clearly envisages a cyclic pattern in the fact that epochs of frequent eruptions appear regularly alternated with periods of less frequent events. It ought to be emphasized that such a pattern is cyclic and not periodic, in the fact that the duration of every cycle is different, in general, for different cycles. It is then reasonable to speculate that the duration of a cycle ought to be inversely proportional to the intensity of the heat supply to that volcano. Thus, it is possible to plot the time variation of the relative change of such a heat supply.

The model here considered ought to imply that such a time variation of the prime source of volcanic heat is synchronous for different volcanoes, either from the same or from different areas of the globe. In fact, it has to be expected that such a time modulation is correlated on the planetary scale with solar activity. Gregori (1990) and Gregori et al. (1992) investigated such an effect from A.D. 1550 for Etna and Vesuvius. They found synchronism between the two volcanoes, and also a remarkable clear correlation between the time variation of solar activity and their heat supply. The most striking result, however, was the absence of any time shift between solar activity and heat supply, a fact that finally lead to the con- 


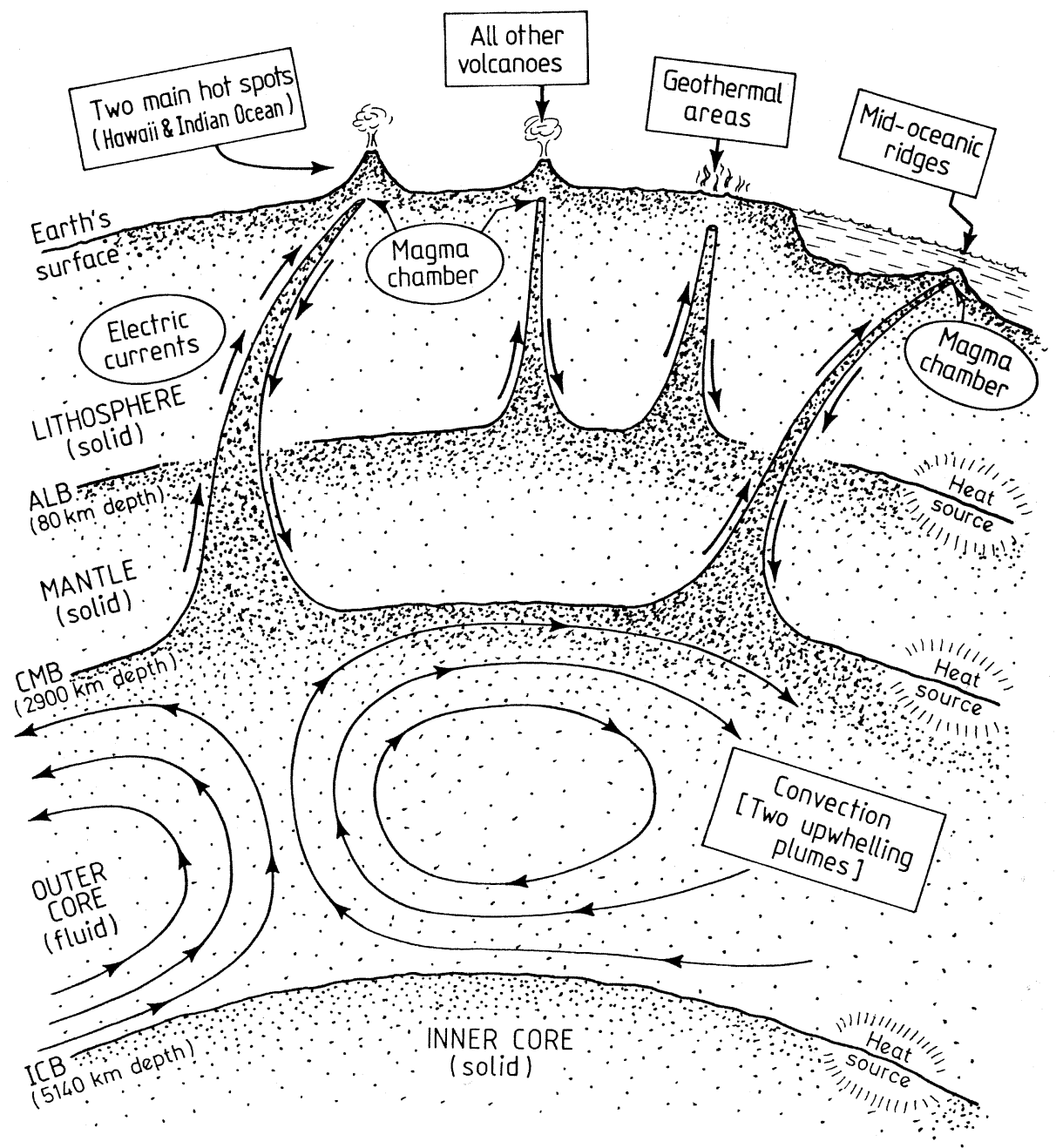

Fig. 3. Three shells of electric currents are expected to exist inside the Earth, located, respectively, at the inner-core boundary (ICB), at the core-mantle boundary (CMB), and at the asthenosphere-lithosphere boundary (ALB). Electric currents tend to expand in space as much as possible (by the Hamilton variational principle). They «push» upward. Wherever there is a bump on either one of such surfaces, a higher concentration of electric currents produces a local increase in heat (and eventually melting) and a higher electrical conductivity $\sigma$. Hence, an additional outward expansion of the electric currents occurs in the region of higher $\sigma$. Thus, currents push upward resulting into actual «pipelines» or «dykes», composed of a material that has a larger $\sigma$, compared with their respective surrounding medium. Such «pipelines» or «dykes» are therefore elongated towards the Earth's surface, although in principle they imply no transport of matter. Eventually, on some locations they reach a very shallow depth, e.g. up to the magma chamber of a volcano. Electric currents dissipate their energy, as Joule's heating, directly at their most shallow depth, where $\sigma$ becomes comparatively lower. Therefore, there are volcanoes that are «rooted» either on the ALB, or on the CMB (while the heat produced on the ICB can be promptly transported up to the CMB simply by convection within the fluid outer core). This sketch is out-of-scale. 
clusion of the role of the afore-mentioned «electric soldering iron» mechanism. Moreover, concerning Etna, a correlation was found between an apparent decrease in its heat supply between the I century B.C. and the I century A.D., and a simultaneous decrease in the solar activity. All ancient solar activity data were taken from the estimates by Stuiver et al. based on the ${ }^{14} \mathrm{C}$ concentration in historical and archaeological wood. The general trend of the volcanic heat sources to increase from $\sim$ A.D. 1550 to the present seems to be in fair agreement with the transition from the Little Ice Age to the present climate (Gregori, 1991).

A recent investigation (Gregori et al., 1994) deals with this same analysis carried out over additional 36 volcanoes, i.e. over all volcanoes of the world for which the catalogue by the Smithsonian Institution reports at least 30 historical eruptions. Almost all of such 36 volcanoes result fairly consistent with the hypothesis of synchronism with Etna's and confirm the expectation of the model here proposed.

The Imbò analysis also provided a statistical forecast of the next eruption of Vesuvius (Gregori, 1993a). In fact, when applying the Imbò's algorithm to the $\sim 2000$ year time series of the historical eruptions of Vesuvius (the wealthiest such series available in addition to Etna's), a regular pattern was found. It appears reasonable therefore to envisage that the present quiescent period of Vesuvius, that began in A.D. 1944, should conclude (due to the previous evidence of two similar long «main» cycles, lasting each one almost $\sim 7$ centuries) sometime between A.D. 2015 and 2029. Moreover, when considering also the morphology of the eruptions, it has to be concluded that a most violent and dramatic event, a sub-Plinian eruption, ought to be expected.

\section{Geomagnetic secular variation, westward drift, field reversals and excursions, magma floods and large igneous provinces, palaeoclimatology}

The geomagnetic SV helps in envisaging that mid-ocean ridges ought to be associated with «dikes» rising from the CMB. Also the anomalies of the westward drift can be explained in terms of such a model (Gregori, 1993). The investigation of the «foci» and of the «epicentres» of the SV, and of their respective motions (the «epicentres» were extensively investigated in the last several years by the Russian school) could be indicative of a time varying geometry, deviating from spherical symmetry, of the surfaces having equal electrical conductivity within the deep Earth's interior. Inferences can perhaps be given also about the relations between the geomagnetic field, gravity, and the anomalies of the Earth's spin rate (in progress).

An inference of the dynamo model here considered deals with the field reversals (FR): according to such a model, it appears very unlikely (if possible at all) that an FR can be triggered by an endogenous process. Rather, it ought to be triggered by a variation of the solar wind. This possibility can be conceptually clarified by considering a very simple model, that is not realistic per se, but that explains the principle idea of the process that can produce a FR in actual reality (fig. 4a-e).

Therefore, one must expect a correlation (either with or without a relative time shift) between FR's and volcanic activity, and it should be a synchronous effect all over the world. The frequency of FR's (their number per unit time) ought to be correlated with the total volume of magma produced by the hot spots. The great volcanic crises (magma floods originating the large igneous provinces and the continental flood basalts) ought thus to be explained in terms of the geomagnetic field (in fact, it has been well known for a long time that sometimes such events apparently occurred almost at the same time in different areas of the world).

Their investigation is presently in progress (in cooperation with Dong Wenjie). The data base being analyzed at present is the output of the Hawaiian hot spot during the last $\sim 70 \mathrm{Ma}$. A few other hot spots are also available, although with fewer details: they will be investigated for getting comparable determinations of the same parameters from similar data series from different parts of the world. In general, a time-shift of $\sim 12.3 \mathrm{Ma}$ between FR and 

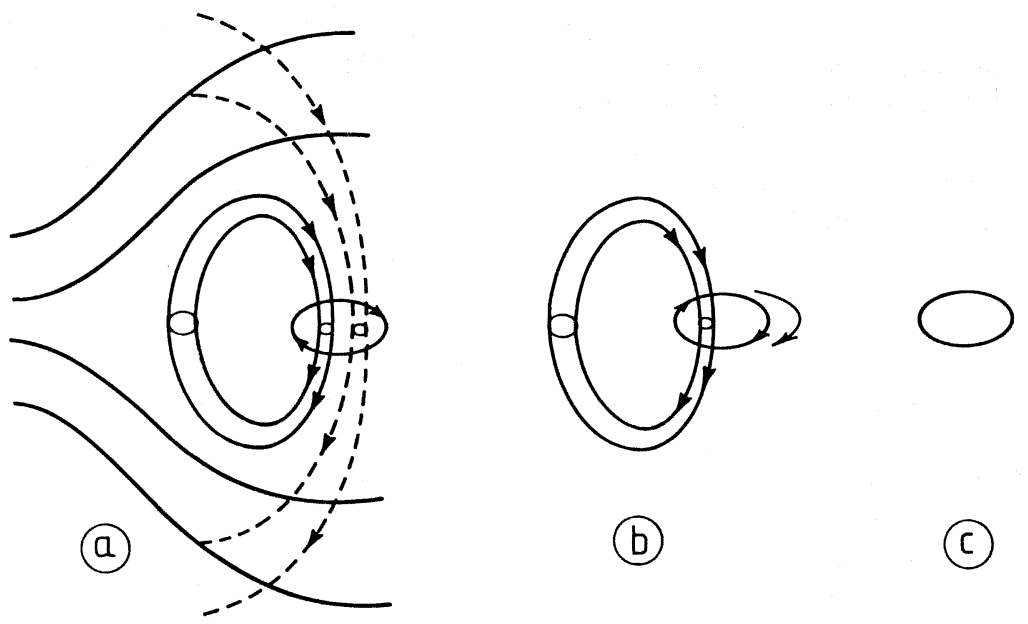

(b)
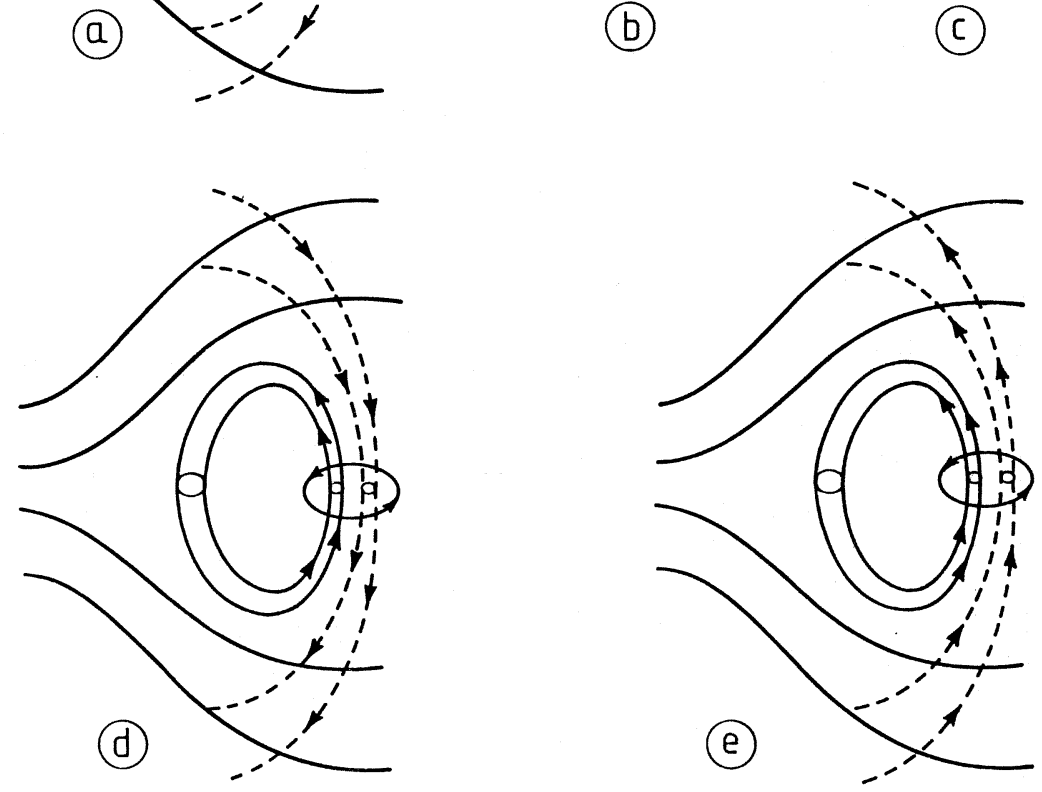

Fig. 4a-e. This sketch represents an ideal experiment aimed to show how geomagnetic field reversals and excursions can occur. The system here considered is a simplified model, by which the Earth is represented by a simple loop (made by an almost perfect conductor) with an electric current flowing inside it. The solar wind blows against it, and some magnetic flux of the interplanetary magnetic field is linked by the loop (a). Suppose the solar wind, hence also its embedded field, is switched off. By induction, an extra current is thus generated inside the loop: the geomagnetic field is strengthened (b). Suppose that the loop remains without solar wind for some time, at least as long as needed for allowing its electric currents to decay (c). Then, switch on anew the solar wind, and its embedded magnetic field as well. Let us suppose that the flux, that is linked by the loop, of such a magnetic field has the same sign that it had prior to the switching off. Then, the newly generated geomagnetic field is opposite compared to the original field: therefore, a field reversal occurred (d). In contrast, if the afore-mentioned flux has an opposite sign with respect to its original value prior to switching off, the newly generated field has the same direction as before: a geomagnetic excursion happened (e). In actual reality, the switching off, and on, of the solar wind could be originated by the encounter, by the solar system, of an interstellar hydrogen cloud, capable of compressing the solar wind inside the orbit of the Earth (see text). 
magma output is the apparent time needed for «opening» or «widening» the «pipeline» by the «electric soldering iron». But, the phenomenon appears much more complicated when considering also its minor details. Also some different time shifts can occur, and there is a smaller and almost simultaneous effect between FR and volcanoes. Also palaeoclimatology seems to be in close agreement with FR and volcanic data. The trend of vulcanism in the future - 12.3 Ma can also be predicted, on the basis of the information on the frequency of the most recent FR's. The general future trend of vulcanism could be towards an increase, that, may imply (on the time scale of a few million years) even a natural general increase in the global climatic temperature and sea level (investigation in progress).

Finally, some suggestions are reported within the literature about the eventual possibility that the Sun, during its motion around the galactic centre, encounters clouds of gas capable of compressing the solar wind inside the Earth's orbit. McKay and Thomas (1978) investigated the effects on the Earth's atmosphere, of the direct interaction of such an interstellar gas. They concluded that a likely effect is the disappearance of the ionospheric $F$ layer, the generation of mesospheric water clouds, and the increase in albedo, the entire effect possibly resulting in triggering a glaciation. But, the dynamo model here proposed implies, as above and in addition to such effects, also the triggering either of a FR or of a geomagnetic excursion, hence substantial changes in the volcanic output, and consequent implications on the global climate (in progress). McElhinny (1971) and Negi and Tiwari (1983) also suggested that the spectrum of occurrence of FR's has an apparent main period component of $\sim 280 \mathrm{Ma}$, that appears to be in close agreement with the known period of revolution of the Sun around the centre of the galaxy. That is, also this feature seems to support the speculation that the Sun, more or less periodically, encounters gas clouds into interstellar space, that trigger either FR's or geomagnetic excursions. Further investigations are in progress (in cooperation with Dong Wenjie).

\section{Global climate change. The role of volcanoes and/or of geothermal heat flow}

A time-varying amount of heat, that must be released from the Earth, implies a time-varying degassing from soil, and/or from seas and oceans, hence a time-varying atmospheric chemistry, greenhouse effect, ozone layer, etc. Basically, three classes of phenomena ought to be distinguished: 1) explosive vulcanism; 2) «quiet», or «normal», or «standard» vulcanism; and 3) geothermal heat flow.

The large volcanic explosions are the best investigated phenomenon. The energy associated with gas injection by explosion is $\sim 1 \%$ of the total thermal energy of the eruption. However, the relationship between the frequency of such events, and the time constant for the decay of their effects is not yet clear.

The role of standard vulcanism, including fumarole activity, is a present challenge. The amount of gas emissions from the slopes of a volcano, when they are integrated over the entire mountain, are comparable with the gas emissions from its crater (S. Lombardi, private communication). This implies that the well known $\mathrm{CO}_{2}$ data series from the Mauna Loa Observatory cannot be considered representative of the average $\mathrm{CO}_{2}$ concentration of anthropic origin within the atmosphere. Rather, it is representative, maybe, only of the time change of the average $\mathrm{CO}_{2}$ emissions from the Hawaiian hot spot. That is, there is a need to monitor the concentration of the minor atmospheric constituents, whatever their origin may be, i.e. either natural or anthropic, mainly from high altitude observatories in order to avoid the disturbance of the absorption by the lowest levels of the atmosphere, and within a network of recording sites suitably located all over the globe.

When considering the volcanic input into the atmosphere (either explosive or standard), attention is normally devoted to the greenhouse gases. All such gases, however, are a permanent feature of the atmosphere, being originated either from the Earth's interior, or from the biosphere, or by anthropic action. There is need, rather, to recognize some tracking gases, 
e.g. some gases that may apparently be unimportant from the climatic viewpoint, but that are emitted only by volcanoes and not by other sources, and that have some limited residencetime within the atmosphere, in order to allow for a suitable time resolution in the monitoring of the phenomena originating their injection into the atmosphere.

An even more difficult problem is the possible role of the degassing from the Earth, that is associated with a time-variation of the geothermal heat flow. Some preliminary discussion is given in Gregori (1991). A few additional hints are as follows. On the time range of several decades, the correlation (Friis-Christensen and Lassen, 1991) between the variation of the total duration of the 11 year cycle of the Sun, and the global climatic temperature, is a clear support for the model here proposed. On the much shorter (yearly) time range, the «warm pool» formation in the Pacific Ocean supports the possibility of such a mechanism. That is, the isocontour lines of the ocean water that is warmer than $28{ }^{\circ} \mathrm{C}$ correlate with the geographic distribution of the optically-thin cirrus clouds, and envisage that a negligib ${ }^{1} \mathrm{c}$ trigger, by means of a very small increase in the water temperature, can start a self-amplifying process, by which the increased greenho' se effect produces an even larger increase in the water temperature. Such a self-amplification stops only when the greater albedo forbids additional absorption of solar radiation (see Ramanathan and Collins, 1991, and Prabhakara et al., 1993, 1993a). But, also the role of the biosphere can be crucial. In fact, a very modest increase in vegetation in a desert appears to be capable even of increasing the rainfall in a wider area, and such an effect should not occur by simple evapotranspiration, but should be due to the change in the thermal inertia of soil (Otterman, 1993 and references therein). Moreover, rainfall seems to be triggered, or controlled, by a slight variation of the local heat flow from the ground (Dong et al., 1990, Dong and Tang, 1992, Tang and Dong, 1993, and Tang et al., 1993). This entire topic needs, however, much harder thinking (in progress).

In any case, a crucial role is played by the very complex phenomena that control the heat transfer into the atmosphere from either soil, or biosphere, or sea or ocean water, or ice or snow (with or without underlying permafrost).

A related item is the interpretation of the "geotherms», i.e. of the temperature profile vs. depth within drill holes, either in permafrost or in other areas (e.g. Gregori and Martellucci, 1992). Geotherms are usually measured for investigating the heat flow from ground. Three different viewpoints, however, compete with each other. 1) One viewpoint is concerned with the record of the geothermal flux per se, that considers only heat-conduction through soil, and that is the concern of the geothermologist. 2) Another viewpoint is related to the heat transport by means of advection by underground fluids (water, gases, oil, etc.); it is the normal concern of the hydrologist. 3) The third viewpoint is concerned with the observed heat flow at the Earth's surface, a parameter that is important for the meteorologist.

The interpretation of geotherms in permafrost areas (mainly by A. Lachenbruch), or also in other areas $(\mathrm{H}$. Pollack and others), is important to obtain a record of the change of the global climate temperature during the last century. This could even be, however, a way of recording the time variation of the heat generation within the Earth's interior, provided that the source be suitably shallow, as otherwise the very low heat conductivity damps off the whole signal, before it reaches the Earth's surface.

Summarizing, one is concerned with either direct or indirect observational evidence that spans different time scales. There are phenomena occurring either in real time or in the yearly time range, that can be monitored either by satellite or by ground-based observatories. There are records dating back several decades (instrumental period), or the proxy and/or historical data records (dating back several centuries to $\mathrm{a} \sim 10^{4}$ years). Ice cores and sediments give information in the few $\sim 10^{5}$ year range, while ocean floor expansion, hot spot trails, large igneous provinces, palaeoclimatology, extinctions in the biosphere, etc. span the range of several $\sim 10^{7}$ years, to a few $\sim 10^{8}$ years. The Sun's motion around the galactic centre has a period of $\sim 280 \mathrm{Ma}$. Throughout 
such time ranges, a mutual interaction existed between different direct, or indirect, physical quantities recorded by Earth sciences, including the complex, difficult, and often neglected role of the changing biosphere.

In this respect, dealing with the historic time scale, Gregori et al. (1994a) were concerned with the floods and anomalous climatic events in North-Western Italy (an almost $\sim 9$ century series for the Tanaro valley). They also used the $\sim 22$ century time series of the Tiber floods, and the $\sim 4$ century time series of the volcanic dust veil index (DVI). The apparent periodicities reported in the literature for varves (lake sediments) approximately range, as a thumb rule, in the range of $\sim 22, \sim 44$, and $\sim 88$ years (every figure plus or minus a few years). It appears reasonable to guess that also volcanic eruptions respond to a $\sim 44$ year cycle, that the consequent degassing and global climate-control have a similar apparent cycle, and finally that also floods and climatic anomalies in the Po valley reflect such an apparently cyclic trend. Such a guess was also used for a tentative and successful prediction.

\section{Implications on the deep structure of the Earth}

The heat transport across the deep layers of the Earth is a crucial aspect for all the forgoing items, as well as for all previously proposed models for the deep Earth's interior. In particular, a most important point is concerned with mantle convection.

The models proposed in the literature span a variety of distinctions, more or less directly reckoning to a few leading schemes. The best known is plate tectonics, but one should also consider the Earth's expansion hypothesis, or even the apparently almost abandoned «fixistic» viewpoint, etc. Maybe, a final definite assessment sensu stricto cannot be attained either in favour or against either interpretation. The correct approach is rather to attempt to understand what supports either viewpoint, or what can be measured for additional checks and inferences, or disproofs.
Concerning plate tectonics, a few paradoxes are well known. For example, one is concerned with the balance between ridges and subduction zones, and in particular with Antarctica, that lies almost completely inside a circle of ridges, with no subduction zone. Another poorly understood piece of evidence is the occurrence of island arcs, that exist only on the western sides of either the Pacific or the Atlantic Ocean (but not in the Indian Ocean); moreover, trenches close to island arcs reveal a tensile stress rather than compression. Another paradox deals with the «Polfluchtkraft» or Eötvös force, i.e. suppose that plates float over a fluid (Newtonian) mantle, and consider the effect of the different application-point of the centrifugal force and of the Archimedean buoyancy force, by which continents should move towards the equator. Three pieces of evidence, however, seem to deny such a conclusion: 1) Antarctica slid towards the South pole several tens of millions of years ago; 2) the distance between South America and Antarctica definitely appears to have shrunk, rather than enlarged, during the geologic past; and 3) Alaska was at a low latitude prior to sliding up to its present location. Therefore, in the final analysis, such effects seem to contradict even the very existence of a Newtonian fluid in the mantle, i.e. even the isostasy hypothesis. In contrast, continental plates apparently behave like rocky blocks that slide over a rigid inner Earth, almost like when people play the «curling» game in Scotland.

Concerning the convection within the mantle, the afore-mentioned volcanic cycle evidence implies (on the historical time scale) an almost «instantaneous» heat transport from the source of volcanoes up to their manifestation on the Earth's surface, requiring by this the existence of the «electric soldering iron that pushes against a block of ice». On the other hand, on the time scale of palaeontology, the afore-mentioned time delay of $\sim 12.3 \mathrm{Ma}, \mathrm{ob}-$ served between FR's and magma production rate, envisages a well defined time lag for allowing the «electric soldering iron» to penetrate into the mantle and lithosphere.

Mantle convection is generally reported as occurring at a speed of one to a few centime- 
tres per year. A heat source active at the CMB, in order to release heat from the Earth's surface, must therefore cross a layer $\sim 2900 \mathrm{~km}$ thick, that takes $\sim 290 \mathrm{Ma}$ (at a $\sim 1 \mathrm{~cm} /$ year speed). This is at least one order of magnitude longer than the observed time-shift between FR's and magma yield. That is, if mantle convection (conceived either as a steady, or even as an intermittent, phenomenon) actually exists, it is concerned with a time scale that appears to be much longer than the processes considered by the model here proposed.

Differently stated, from such a viewpoint, when considering any one given observational data set, one should first distinguish between phenomena occurring on different time scales. On the very short (i.e. present-day, or historical) time scale, one observes the eventual modulation of the output, that uses the already existing channels for heat transport. But, if such already available channels are insufficient to ensure the thermal balance of the Earth, other phenomena must unavoidably start and evolve on the longer time scale(s). Hence, on some intermediate time scale(s), one should observe other effects related to the slow changes of some basic features (in the present case one observes a time change of the cross section, or of the heat transport efficiency, of the «pipelines»). On some even longer time scale, one can eventually observe, perhaps, also other phenomena, such as mantle convection.

All Earth models, however, unavoidably rely on a great deal of speculation and arbitrariness. In this same respect, the study of the history of scientific ideas appears to be crucial: we need to understand how far any given theory or model has sound observational basis at present, and how far it is, rather, the result of «opinion» science. This recalls the similar classical example of the epicycles, when, rather than adopting the heliocentric hypothesis, they preferred to introduce such complicated concepts. This matter is akin to the well known classical philosophical debate about historiography (e.g. Kragh, 1987), by which the concern is whether history should be an objective and very detailed report by a chronicler, or should rather be the result of a synthesis by the histo- rian, who makes a selection of facts by choosing only a few of the most relevant events that he believes can be significant to prove his own interpretation of history. This point is basically related to the intrinsic difference between a chronicler and a historian. In the case of science, the question is whether the history of science should be only a search for, and a collection of, some curiosities about the life and ideas of some past scientists, or whether it should be a re-interpretation of the history of the past evolution of ideas. In such a second case, it is not important that the historian scans some as yet unpublished documentation. Rather, he can rely on what is already available and well known, but he re-interprets it for a better understanding of the ideas, models, and philosophy, for recognizing what is «opinion» science and what is proven by sound observational evidence (in physics, this is like the difference between a theoretician and an experimentalist). For example, both the hypotheses of isostasy and of mantle convection can be conceived as being daughters of the philosophy prevailing at the end of the past century, by which the Earth was conceived as a fluid ball with a very thin solid crust floating over it. From such a viewpoint, the Newtonian fluid hypothesis could even be conceived as a mathematical fiction, much like the epicycles, aimed at maintaining an old philosophy. On the other hand, mantle convection is generally also believed to be a fundamental need to explain heat transport through the Earth, as conduction alone is definitely unviable. As a matter of fact, the experimental evidence proves that internal heat exists, for feeding both volcanoes and geothermal flow, and for being perhaps associated with the endogenous processes supplying geodynamics. In contrast, however, within the model here discussed, a large fraction of the endogenous heat ought to be transported most simply by electric currents, that release the largest amount of heating only at some very shallow depth underground. Hence, from such a viewpoint, there could be even no strict need for appealing to mantle convection. On the other hand, at present there is no sound reason why such a conclusion ought to be considered either wrong or correct. The history of 
science is made of concepts, hence unavoidably also of «opinions». The most difficult task of every scientist is to succeed in distinguishing the facts that are actually proved by observations, from the «generally agreed» models.

\section{REFERENCES}

Dong, W.J., M.C. TANG and J. ZHANG (1990): Approach on the underground suddenly warming relative to shortterm climatic variations, Plateau Meteorol., 9 (4), 364376.

DONG, W.J. and M.C. TANG (1992): Preliminary results of mean soil heat flow calculated by soil temperature data observed at meteorological stations, Plateau Meteorol., 11 (2), 115-125.

FriIs-Christensen, E. and K. LASSEN (1991): Length of the solar cycle: an indicator of solar activity closely associated with climate, Science, 254, 698-700.

GREGORI, G.P. (1990): La ricerca di ciclicità e l'algoritmo di Imbò - Applicazione alle serie storiche delle eruzioni dell'Etna e del Vesuvio in Proceedings of "Convegno del Progetto Strategico Clima, Ambiente e Territorio nel Mezzogiorno», Potenza (in press).

GREGORI, G.P. (1991): Anthropic control of the natural long-term climatic variations: proposal for a discussion on greenhouse effect controlled by geothermal heat flow, in Proceedings of the International School on Plasma Physics «Piero Caldirola» on Controlled Active Global Experiments (CAGE), Varenna (Como, Italy), September 5-12, 1990, edited by E. SINDONI and A.Y. Wong (Editrice Compositori, SIF, Bologna), 353360.

GREGORI, G.P. (1993): Geo-electromagnetism and geodynamics: «corona discharge» from volcanic and geothermal areas, Phys. Earth Planet. Inter., 77, 39-63.

GREGORI, G.P. (1993a): The next eruption of Somma-Vesuvius, invited paper in The Earth and the Universe, Festschrift in Honour of Prof. Hans-Jürgen Treder, edited by W. SCHRÖDER (Bremen, Science Edition) 191213.

GregorI, G.P. and S. MARTELluCCI (1992): Underground record of global climate change, in 4th Workshop Italian Research on Antarctic Atmosphere, Porano (Terni, Italy) October 21-23, 1991, edited by M. CoLACino, G. Giovannelli and L. Stefanutti, Conference Proceedings (Società Italiana di Fisica, Bologna), 35, 279-293.

Gregori, G.P., V. BAnzon, R. LeOnardi and G. DE FRANCESCHI (1992): Geomagnetic activity vs. volcanic cycles, and their forecasting. Application to Etna and Vesuvius, in Solar Terrestrial Variability and Global Change, edited by W. SCHRÖDER and J.P. LEGRAND (Interdivisional Commission on History of IAGA, Bremen-Roennebeck), 188-222.

GREGORI, G.P., V. BANZON and R. LEONARDI (1994): Volca- noes and Joule's heating from the dynamo. The time pattern: synchronism of the time variation of the source, and volcanic cycles, in Geophysics: past achievements and future challenges, edited by W. SCHRÖDER and M. COLACINO (Interdivisional Commission on History of IAGA, Bremen-Roennebeck), 152191.

Gregori, G.P., V. BAnZon, M. Colacino, G. DE Franceschi, L. Diodato, M.P. PAVEse and R. SANTOleri (1994a): Floods, and volcanic activity indices, in Geophysics: past achievements and future challenges, edited by W. SCHRÖDER and M. COLACINO (Interdivisional Commission on History of IAGA, Bremen-Roennebeck), 109133.

KraGH, H. (1987): An Introduction to the Historiography of Science (Cambridge Univ. Press, Cambridge), Italian translation published in 1990 by Zanichelli, Bologna, pp. 256.

McELhinny, M. W. (1971): Geomagnetic reversals during the Phanerozoic, Science, 172, 157-159.

MCKAY, C.P. and G. E. Thomas (1978): Consequences of a past encounter of the Earth with an interstellar cloud, Geophys. Res. Lett., 5, (3), 215-218.

NEGI, J.G. and R.K. TIWARI (1983): Matching long term periodicities of geomagnetic reversals and galactic motions of the solar system, Geophys. Res. Lett., 10 (8), 713-716.

OtTerman, J., M.D. Novak and D.O'C. Starr (1993): Turbulent heat transfer from a sparsely vegetated surface: two-component representation, Boundary Layer Meteorol., 64, 409-420.

Prabhakara, C., D.P. Kratz, J.-M. Yoo, G. Dalu and A. VERNEKAR (1993): Optically thin cirrus clouds: radiative impact on the warm pool, J. Quant. Spectrosc. Radiat. Transfer, 49 (5), 467-483.

Prabhakara, C., J.-M. Yoo, D.P. Kratz and G. Dalu (1993a): Boundary layer stratus clouds: inferred from satellite infrared spectral measurements over oceans, J. Quant. Spectrosc. Radiat. Transfer, 49 (6), 599607.

Ramanathan, V. and W. Collins (1991): Thermodynamic regulation of ocean warming by cirrus clouds deduced from observations of the 1987 El Nino, Nature, 351, 27-32.

Ronov, A.B. (1975): The Earth's Sedimentary Shell. Quantitative Patterns of its Structure, Compositions, and Evolution (the 20th V. I. Vernadskiy Lecture), The American Geological Institute, AGI reprint series V, pp. 80.

TANG, M.C. and W.J. Dong (1993): Advances in Studies on «Earth-Atmosphere Coupling» (comprehensive review), preprint, pp. 10.

TANG, M.C., W.J. Dong, J. ZHANG and L. YANG (1993): Correlation analysis of the runoff of the rivers and the seism in China, Sci. in China, ser. B (Sci. Sin.), 36 (5), 630-640.

WILSON, M. (1989): Igneous Petrogenesis. A Global Tectonic Approach (Unwin Hyman, London, etc.), pp. 466. 